\title{
ON MATRICES WHOSE CHARACTERISTIC EQUATIONS ARE IDENTICAL
}

\author{
W. V. PARKER
}

In a previous paper [ 1$]^{1}$ it was shown that if $A$ and $C$ are matrices such that $A C A=0$ and $B$ is an arbitrary matrix, then $A B$ and $A(B+C)$ have the same characteristic equation. It is the purpose of this note to prove two theorems, each of which gives the above result as a special case.

TheOREM 1. Let $A$ be an $n \times m$ matrix of rank $r<n$ and let $C$ be an $m \times n$ matrix such that $A C A=k A$ ( $k$ a scalar). If $B$ is an $m \times n$ matrix, the characteristic equation of $A B$ is $x^{n-r} \phi(x)=0$ and the characteristic equation of $A(B+C)$ is $x^{n-r} \phi(x-k)=0$.

Let $P$ and $Q$ be nonsingular matrices such that

$$
P A Q=\left(\begin{array}{ll}
I_{r} & 0 \\
0 & 0
\end{array}\right), Q^{-1} B P^{-1}=\left(\begin{array}{ll}
B_{1} & B_{2} \\
B_{3} & B_{4}
\end{array}\right), \text { and } Q^{-1} C P^{-1}=\left(\begin{array}{ll}
C_{1} & C_{2} \\
C_{3} & C_{4}
\end{array}\right)
$$

where $B_{1}$ and $C_{1}$ are square matrices of order $r$. Then since $A C A=k A$,

$$
P A C A Q=\left(\begin{array}{ll}
I_{r} & 0 \\
0 & 0
\end{array}\right)\left(\begin{array}{ll}
C_{1} & C_{2} \\
C_{3} & C_{4}
\end{array}\right)\left(\begin{array}{ll}
I_{r} & 0 \\
0 & 0
\end{array}\right)=\left(\begin{array}{ll}
C_{1} & 0 \\
0 & 0
\end{array}\right)=\left(\begin{array}{cc}
k I_{r} & 0 \\
0 & 0
\end{array}\right)
$$

and hence $C_{1}=k I_{\text {r. }}$.

Also

$$
P A C P^{-1}=\left(\begin{array}{ll}
I_{r} & 0 \\
0 & 0
\end{array}\right)\left(\begin{array}{ll}
C_{1} & C_{2} \\
C_{8} & C_{4}
\end{array}\right)=\left(\begin{array}{cc}
k I_{r} & C_{2} \\
0 & 0
\end{array}\right)
$$

and

$$
P A B P^{-1}=\left(\begin{array}{ll}
I_{r} & 0 \\
0 & 0
\end{array}\right)\left(\begin{array}{cc}
B_{1} & B_{2} \\
B_{3} & B_{1}
\end{array}\right)=\left(\begin{array}{cc}
B_{1} & B_{2} \\
0 & 0
\end{array}\right)
$$

and hence

$$
P(A B+A C) P^{-1}=\left(\begin{array}{cc}
B_{1}+k I_{r} & B_{2}+C_{2} \\
0 & 0
\end{array}\right) .
$$

Presented to the Society, September 2, 1949; received by the editors February 16, 1949 and, in revised form, May 19, 1949.

1 Numbers in brackets refer to the references cited at the end of the paper. 
It follows that the characteristic equation of $A B$ is $x^{n-r} \phi(x)=0$ and the characteristic equation of $A(B+C)$ is $x^{n-r} \phi(x-k)=0$ where $\phi(x)=0$ is the characteristic equation of $B_{1}$. The result of the previous paper follows if $k=0$.

In case $k \neq 0$ it may as well be assumed that $k=1$ so that $A C A=A$ Set $U=A B, E=A C$ and $V=U+E$. Then $E^{2}=E, E U=U$, and $E V$ $=V$. Hence $V^{\cdot} U=(U+1)^{\bullet} U$ and $U^{\bullet} V=(V-1)^{\bullet} V$ so that $f(V) U$ $=f(U+1) U$ and $f(U) V=f(V-1) V$ for all polynomials $f(x)$. Let $g(x)$ be the minimum function of $U$ and $h(x)$ the minimum function of $V$. Then $g(V-1) V=g(U) V=0$ and $h(U+1) U=h(V) U=0$ so that $h(x) \mid x g(x-1)$ and $g(x) \mid x h(x+1)$. Hence the minimum. functions of $U$ and $V$ satisfy one of the four relations:
(1) $h(x)=g(x-1)$;
(2) $(x-1) h(x)=x g(x-1)$;
(3) $(x-1) h(x)=g(x-1)$;
(4) $h(x)=x g(x-1)$.

That all four relations are actually possible may be shown by examples.

THEOREM 2. If $M$ and $N$ are square matrices such that $N M$ (or $M N)=N^{2}=0$, then $M$ and $M+N$ have the same characteristic equation.

Since $N^{2}=0, N$ is similar to

$$
\left(\begin{array}{lll}
0 r & I_{r} & 0 \\
0 r & 0 & 0 \\
0 & 0 & 0
\end{array}\right)
$$

where $r$ is the rank of $N$. There is no loss of generality in assuming that $N$ is in this form. Then since $N M=0$

$$
M=\left(\begin{array}{lll}
M_{1} & M_{2} & M_{3} \\
0_{r} & 0_{r} & 0 \\
M_{4} & M_{5} & M_{6}
\end{array}\right)
$$

where $M_{1}$ and $M_{2}$ are square matrices of order $r$. Any principal minor of $M$ which contains elements of $M_{2}$ also contains a row of zeros from rows $r+1$ to $2 r$. Hence the characteristic equation of $M$ is independent of the elements of $M_{2}$. But $M$ and $M+N$ differ only in the elements of $M_{2}$ and therefore their characteristic equations are identical. If $M N=N^{2}=0$, then $N^{\prime} M^{\prime}=N^{\prime 2}=0$, so that $M^{\prime}$ and $M^{\prime}+N^{\prime}$ have the same characteristic equation. But the characteristic equation of any matrix is the same as that of its transposed matrix and the proof of the theorem is complete. 
If $A C A=0$ then $(A C)(A B)=(A C)^{2}=0$ and hence $A B$ and $A(B+C)$ have the same characteristic equation.

It is well known that if $A$ and $B$ are square matrices, $A B$ and $B A$ have the same characteristic equation and if either $A$ or $B$ is nonsingular, the two products are similar. Roth [2] has pointed out that if both $A$ and $B$ are singular, the products may be similar or not.

With $P$ and $Q$ as defined in Theorem 1

$$
P A B P^{-1}=\left(\begin{array}{ll}
B_{1} & B_{2} \\
0 & 0
\end{array}\right)=\left(\begin{array}{ll}
B_{1} & 0 \\
0 & 0
\end{array}\right)+\left(\begin{array}{ll}
0 & B_{2} \\
0 & 0
\end{array}\right)=M+N_{1}
$$

where $N_{1} M=N_{1}^{2}=0$ and

$$
Q^{-1} B A Q=\left(\begin{array}{ll}
B_{1} & 0 \\
B_{3} & 0
\end{array}\right)=\left(\begin{array}{ll}
B_{1} & 0 \\
0 & 0
\end{array}\right)+\left(\begin{array}{ll}
0 & 0 \\
B_{3} & 0
\end{array}\right)=M+N_{2}
$$

where $M N_{2}=N_{2}^{2}=0$.

Hence

$$
A B=P^{-1} M P+P^{-1} N_{1} P=K+S
$$

where $S A=S^{2}=0$ and

$$
B A=Q M Q^{-1}+Q N_{2} Q^{-1}=L+T
$$

where $A T=T^{2}=0$. Since $K=P^{-1} M P=A B-S$ and $L=Q M Q^{-1}$ $=B A-T$ the following theorem is established.

TheOREM 3. If $A$ and $B$ are square matrices, there exist matrices $S$ and $T$ such that $S A=S^{2}=A T=T^{2}=0$ and such that $A B-S$ is similar to $B A-T$.

\section{BIBLIOGRAPHY}

1. W. V. Parker, On the characteristic equations of certain matrices, Bull. Amer. Math. Soc. vol. 55 (1949) pp. 115-116.

2. W. E. Roth, $A$ theorem on matrices, Amer. Math. Monthly vol. 44 (1937) p. 95.

The University of Georgia 